\title{
QSOs from the variability and proper motion survey in the M 3 field ${ }^{\star}, \star \star$
}

\author{
H. Meusinger ${ }^{1, \star \star}$, R.-D. Scholz ${ }^{2, \star \star \star}$, M. Irwin ${ }^{3}$, and H. Lehmann ${ }^{1, \star \star \star}$ \\ 1 Thüringer Landessternwarte Tautenburg, 07778 Tautenburg, Germany \\ 2 Astrophysikalisches Institut Potsdam, An der Sternwarte 16, 14482 Potsdam, Germany \\ 3 Institute of Astronomy, Madingley Road, Cambridge CB3 1HA, UK
}

Received 5 June 2002 / Accepted 27 June 2002

\begin{abstract}
We present results of the spectroscopical follow-up observations of QSO candidates from a combined variability and proper motion (VPM) survey in a $\sim 10$ square degrees region centered on the globular cluster M 3. The search is based on a large number of digitised Schmidt plates with a time-baseline of three decades. This paper reviews the candidate selection, the follow-up spectroscopy, and general properties of the resulting QSO sample. In total, 175 QSOs and Sey1s were identified among the objects from the VPM survey, with 114 QSOs and 10 Sey1s up to the pre-estimated 90\% completeness limit of the survey at $B_{\lim } \approx 19.7$. The redshift range of the QSOs is $0.4<z<3$. Among the 80 QSO candidates of highest priority we confirm 75 QSOs/Sey1s and 2 NELGs. We present magnitudes, colours, redshifts, and variability indices for all 181 identified QSOs/Sey1s/NELGs and spectra for the 77 QSOs/Sey1s/NELGs from our spectroscopic follow-up observations. The VPM survey uses selection criteria that are not directly relying on the spectral energy distribution of QSOs. It is therefore remarkable that the properties of the VPM QSOs do not significantly differ from those of samples from colour selection or slitless spectroscopy. In particular, we do not detect a substantial number of unusually red QSOs. The total surface density of the brighter QSOs $(17 \leq B \leq 18.5)$ in our search field is found to be by a factor of $\sim 1.8$ larger than that derived from previous optical surveys.
\end{abstract}

Key words. galaxies: active - galaxies: Seyfert - galaxies: statistics - galaxies: quasars: general

\section{Introduction}

The variability and proper motion (VPM) survey is a QSO search project that is based on optical long-term variability and non-detectable proper motions. Variability of flux densities and stationarity of positions are two fundamental properties of quasars, and therefore well suited as selection criteria of a QSO search (e.g., Kron \& Chiu 1981; Hawkins 1983; Majewski et al. 1991; Véron \& Hawkins 1995; Bershady et al. 1998). However, due to the special demands on the

Send offprint requests to: $\mathrm{H}$. Meusinger, e-mail: meus@tls-tautenburg.de

* The lists of QSOs (Tables 3 and 4) are only available in electronic form at the CDS via anonymous ftp to

cdsarc.u-strasbg.fr (130.79.128.5) or via

http://cdsweb.u-strasbg.fr/cgi-bin/qcat?]/A+A/392/851

$\star \star$ Full Fig. 7 is only available in electronic form

http://www . edpsciences.org

$\star \star \star$ Visiting Astronomer, German-Spanish Astronomical Centre, Calar Alto, operated by the Max-Planck-Institute for Astronomy, Heidelberg, jointly with the Spanish National Commission for Astronomy. number and the time-baseline of the available observations such attempts must be limited to comparatively small and confined areas. We performed the VPM technique in two Schmidt fields of $\sim 10$ square degrees each on the basis of a large number of altogether more than 200 digitised Tautenburg Schmidt plates in the $B$ band with a time-baseline of three decades (Meusinger et al. 1997, 2002).

It is not the primary aim of this project to increase the number of known QSOs by an insignificant fraction; the problems of detecting substantial numbers of QSOs have long been overcome. Over the last decade, among others, the Durham/AAT survey (Boyle et al. 1990), the Large Bright Quasar Survey (Hewett et al. 1995), the Edinburgh Quasar Survey (Goldschmidt \& Miller 1998), and the Hamburg/ESO survey (Wisotzki et al. 2000) have been completed. Presently, the 2dF Quasar Survey (Croom et al. 2001) and the Sloan Digital Sky Survey (Schneider et al. 2002) are extremely efficient at identifying very large numbers of quasars. The INT Wide Angle Survey (Sharp et al. 2001) is expected to detect a statistically significant sample of high-redshift quasars. Very deep quasar samples were obtained in the Lockman hole via the X-ray satellite ROSAT 
(Hasinger et al. 1998) and in the optical domain with the Hubble Space Telescope (e.g., Conti et al. 1998), respectively. Further, the VLA FIRST Bright Quasar Survey (e.g., White et al. 2000) will define a radio-selected QSO sample that is competitive in size with current optically selected samples.

Most of the criteria for the selection of QSO candidates rely upon differences in the broad-band spectral energy distribution of QSOs and stars. Despite the large number of QSOs now catalogued, the selection effects of the conventional surveys are not yet fully understood. It is therefore important to perform QSO surveys that are based on different selection methods. The VPM survey does not directly invoke the spectral energy distribution as the primary selection criterion and provides therefore an interesting opportunity to evaluate the selection effects of more conventional optical QSO searches. For instance, a serious question concerns the possible existence of a substantial population of red QSOs. Extinctionreddened QSOs are suggested both from the AGN unification model (e.g., Antonucci 1993; Maiolino 2001) and from the hypothesis of ultra-luminous IR galaxies (ULIRGs) as QSOs in the making (Sanders et al. 1988). The vast majority of catalogued QSOs have uniform spectral energy distributions with a blue continuum and broad absorption lines. Over the last few years, QSOs with extreme red colours have been detected on the basis of their X-ray emission (e.g., Risaliti et al. 2001) or by radio surveys (Webster et al. 1995; Francis et al. 2000; White et al. 2000; Menou et al. 2001; Gregg et al. 2002). The fraction of unusually red objects among the whole QSO population is however unknown. When compared to other optical surveys, the VPM technique has the advantage that it can discover such red QSOs as long as (1.) they are not too faint in the $B$ band and (2.) they are not much less variable than the conventional QSOs.

The VPM survey was started in the high-galactic latitude field around M 3 (Meusinger et al. 1995). Half of this field is covered by the CFHT blue grens survey (e.g., Crampton et al. 1990). The CFHT QSOs could serve as a training set and were used to define the selection thresholds for the VPM survey in such a way that a $\sim 90 \%$ completeness is expected up to $B \approx 19.7$. The strategy, the observational material, and the data reduction for the M3 field were presented in detail in Paper I (Scholz et al. 1997). The procedures and results for the second search field, around M92, are described in a series of papers (Brunzendorf \& Meusinger 2001, 2002; Meusinger \& Brunzendorf 2001, 2002). A brief review of the whole VPM project is given by Meusinger et al. (2002). The present paper presents the QSO sample in the M3 field. In Sect. 2, we briefly discuss the candidate selection. The spectroscopic follow-up observations are described in Sect. 3. Section 4 gives an overview of the properties of the QSO sample. Conclusions are given in Sect. 5. As in the previous papers, we adopt $H_{0}=50 \mathrm{~km} \mathrm{~s}^{-1} \mathrm{Mpc}^{-1}$ and $q_{0}=0$.

\section{QSO candidate selection}

The VPM search is based on indices for star-like image structure, positional stationarity, overall variability, and long-term variability measured on $57 \mathrm{~B}$ plates taken with the Tautenburg

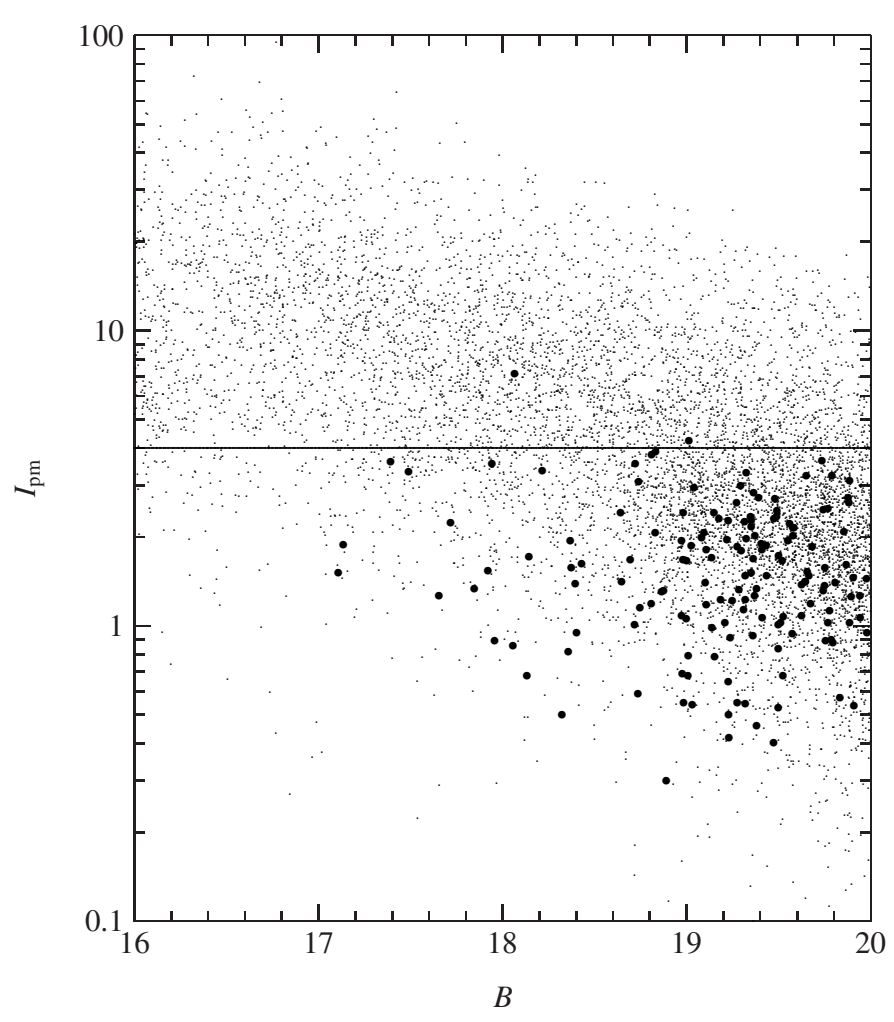

Fig. 1. Proper motion index $I_{\mathrm{pm}}$ as a function of the mean $B$ magnitude for about 7000 star-like objects with $17<B<20$ (small grey dots). The 154 QSOs, Sey1s, and NELGs in this magnitude range are marked as bullets. The horizontal line indicates the proper motion selection threshold.

Schmidt plates between 1964 and 1994, i.e. with a timebaseline of three decades. The $B$ magnitudes given in the present paper are mean magnitudes from this database. The strategy for the QSO candidate selection of the VPM search in the M 3 field and the definitions of the indices are outlined at length in Paper I. Here we review only the basic ideas and describe the modifications in the selection procedures.

The proper motion index, $I_{\mathrm{pm}}$, is expressed simply by the measured proper motion in units of the proper motion error. The overall variability index, $I_{\mathrm{var}}$, is assessed by the deviation of the individual magnitudes about the mean magnitude, and is normalised by the average magnitude scatter for star-like objects in the same magnitude range. Finally, an index for longterm variability, $I_{\text {ltvar }}$, is defined by means of structure function analysis and is computed for all star-like objects with $I_{\mathrm{pm}}<4$ (see below) and $B<20$. The selection thresholds for the indices were derived from the statistics of the previously known QSOs in the field. There are 90 such QSOs identified with star-like objects measured on at least 7 of our $B$ plates. We found that a good compromise between the success rate (i.e., the fraction of candidates that turn out to be QSOs) and the completeness (i.e., the fraction of all QSOs found by the survey) is achieved for the following set of constraints: $I_{\mathrm{pm}}<4, I_{\mathrm{var}}>1.3$, and $I_{\text {ltvar }}>1$.4. (In Paper I, the long-term variability index was denoted $R S_{100}$.) The pre-estimated values for the success rate and the completeness are $90 \%$ and $40 \%$, respectively, for a limiting 
Table 1. Object numbers for the subsamples of QSO candidates with high, medium, or low priority.

\begin{tabular}{llll}
\hline \hline priority class & high & medium & low \\
\hline number of candidates & 80 & 95 & 607 \\
already catalogued & 26 & 17 & 15 \\
newly observed & 54 & 68 & 27 \\
\hline QSOs/Sey1s & 75 & 36 & 20 \\
NELGs & 2 & - & 1 \\
stars & 3 & 49 & 21 \\
\hline
\end{tabular}

magnitude $B_{\lim }=19.7$. (Note that the limiting magnitudes of the individual $B$ plates vary from 19.5 to 21.3 .)

For stationary objects, the probability $p_{\mathrm{pm}}$ to measure a non-zero proper motion follows a Weibull-distribution and depends only on the proper motion index (Brunzendorf \& Meusinger 2001). An object with $I_{\mathrm{pm}} \geq 4$ has a probability of $p \geq 0.9997$ for non-zero proper motion. As illustrated by Fig. 1, the proper motion selection is in particular efficient for brighter magnitudes where the proper motion errors are smaller. For $B<18.5$, the typical proper motion error is about

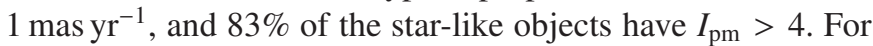
$19<B<20$, on the other hand, the typical proper motion

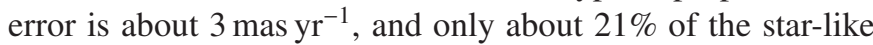
objects have $I_{\mathrm{pm}}>4$. In a flux-limited sample, most of the objects have magnitudes close by the limit. Hence, the proper motion selection appears not very efficient for the whole survey with a limit at $B \approx 19.7$. However, at brighter magnitudes, the number-magnitude relation for QSOs is much steeper than for the foreground stars. This means that the contamination of the variability-selected QSO candidate sample by foreground stars is stronger at brighter magnitudes where the proper motion selection works more efficiently. At fainter magnitudes, the zero proper motion constraint is important in particular for the efficient rejection of nearby variable late-type main sequence stars (see Paper I).

The selection starts with 32700 objects detected on a deep master plate. About 24600 objects were identified on at least two further plates, among them are about 21500 objects with star-like images. A basic object sample for the variability selection is defined by the 12800 star-like objects measured on at least $7 \mathrm{~B}$ plates. After excluding the objects in the crowded cluster region (distance to the centre of M3 less than 24'), this sample is reduced to 8582 objects in total and to 4614 objects in the magnitude range $16.5 \leq B \leq 19.7$, respectively. About $65 \%$ of the objects from this reduced sample are rejected due to the zero proper motion constraint $I_{\mathrm{pm}}<4$. Finally, the variability constraints strongly reduce the candidate sample to a manageable size.

For practical reasons, the candidate sample is devided into three subsamples of different priority. A similar approach was used for the VPM search in the M92 field (cf. Brunzendorf \& Meusinger 2001). However, the variability indices defined there are slightly different from those used in the present study, and the priority classification in the two VPM fields are not completely identical. Here, the priority depends mainly on the variability indices. In addition, the $B$ magnitudes and the crowding of the field (expressed by the distance $d_{\mathrm{c}}$ from the centre of M 3) are taken into account. For all priority classes, star-like objects are considered with $I_{\mathrm{pm}}<4, B=15-19.7$, and $d_{\mathrm{c}}>24$ arcmin. The high-priority subsample consists of the strongly variable objects with $I_{\mathrm{var}}>1.8, I_{\mathrm{ltvar}}>1.8$. The medium-priority subsample contains the objects with smaller variability indices $I_{\mathrm{var}}=1.3-1.8$ and $I_{\text {ltvar }}>1.4-1.8$. In addition, we included the few objects with somewhat higher variabiliy indices $\left(I_{\mathrm{var}}=1.6-1.8\right.$ and $\left.I_{\mathrm{ltvar}}=1.6-1.8\right)$ in the stronger crowded region $d_{\mathrm{c}}=12-24$ arcmin. Finally, the lowpriority subsample comprises the objects having only one of the two variability indices above the threshold (i.e., $I_{\text {var }}>1.3$ or $I_{\text {ltvar }}>1.4$ ). In addition, we consider also objects with $19.7 \leq B \leq 19.8$ and $d_{\mathrm{c}}>12$ arcmin as low-priority candidates if at least one of the two variability indices exceeds the threshold. The variability selection is illustrated by Fig. 2 .

As discussed in Paper I, the $U$ band variability index may serve as an additional selection criterion. In practice, however, the fainter objects are measured on only a small number of $U$ plates. Therefore, the $U$ variability index was invoked only in one case: the QSO No. 51 from Table 3 has insignificant $B$ variability indices but shows significant variability in the $U$ band.

The numbers of selected candidates are 80, 95, and 607, respectively, for the subsamples of high, medium, or low priority (Table 1). It is expected that the fraction of QSOs/Sey $1 \mathrm{~s}$ strongly decreases with decreasing priority. In particular, the low-priority subsample is expected to be strongly contaminated by galactic stars with relatively enhanced photometric errors.

A cross-check of the candidate list against the $\mathrm{NED}^{1}$ (2002, February) yields the identification (identification radius $10 \mathrm{arcsec}$ ) of $57 \mathrm{QSOs} / \mathrm{Sey} 1 \mathrm{~s}$ and one narrow emission line galaxie (NELG) with catalogued redshifts. Over the whole magnitude range, 104 objects with catalogued redshifts $z>0$ were identified (100 QSOs/Sey1s, 4 NELGs). The overwhelming fraction of the QSOs/Sey1s are from the CFHT blue grens survey (e.g., Crampton et al. 1990) which covers approximately half of our survey field.

\section{Spectrosopic follow-up observations}

Spectroscopic follow-up observations have been focussed on the candidates of high or medium priority. Most of the spectra were taken during five observing runs either with the $2.2 \mathrm{~m}$ telescope on Calar Alto or with the Tautenburg $2 \mathrm{~m}$ telescope. An overview of these observation runs is given in Table 2 . In addition, three candidates with uncertain spectroscopic identification were re-observed in July 2001 with CAFOS on Calar Alto; this run is quoted as number 6 in Table 3 . An additional 18 candidates of medium or low priority were proved to be foreground stars during several other campaigns with either the Tautenburg telescope or the Calar Alto $2.2 \mathrm{~m}$ telescope.

1 The NASA/IPAC extragalactic database (NED) is operated by the Jet Propulsion Laboratory, California Institute of Technology, under contract with the National Aeronautics and Space Administration. 


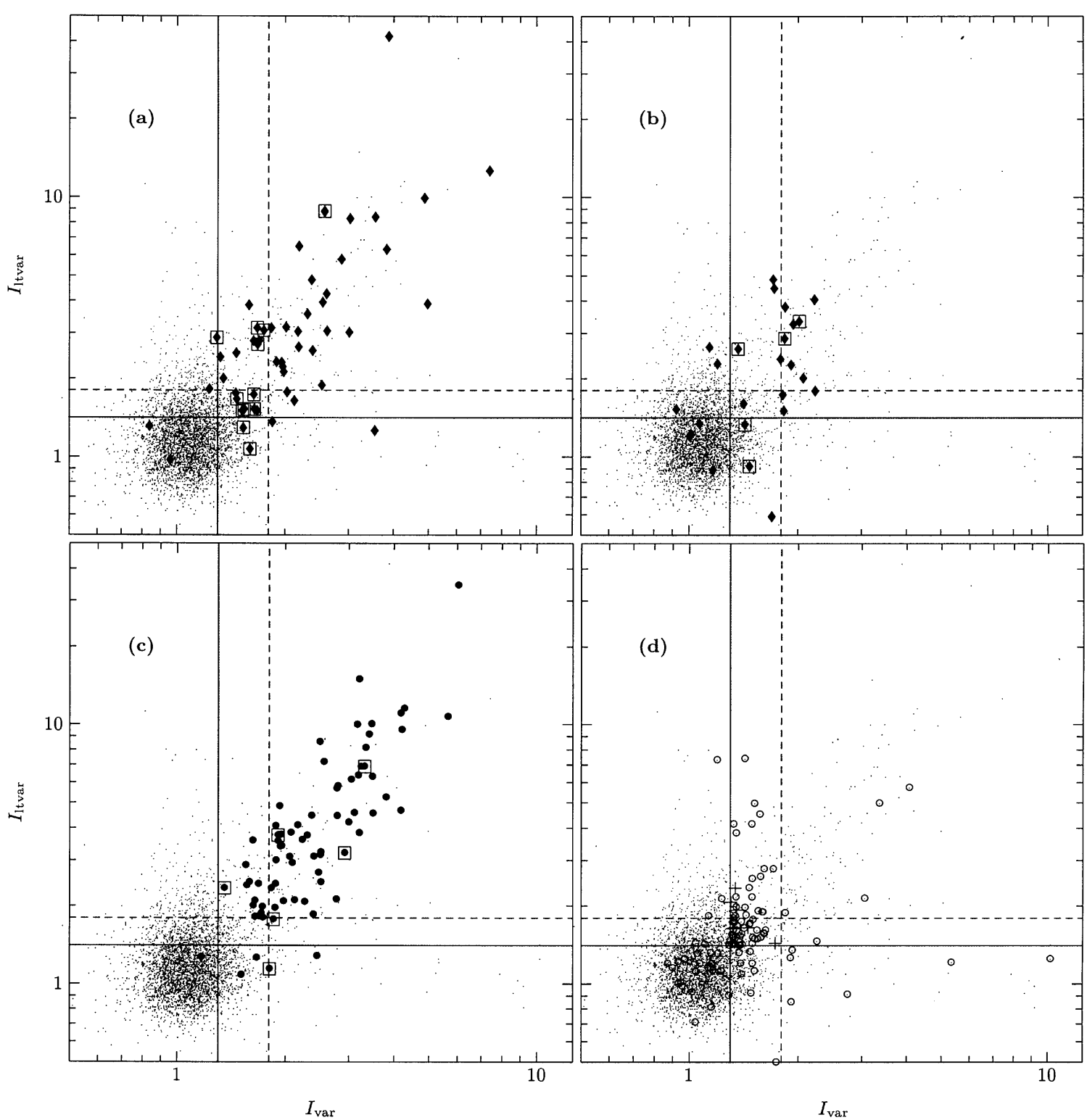

Fig. 2. Long term variability index $I_{\text {ltvar }}$ versus overall variability index $I_{\text {var }}$ for the 4379 star-like objects from the reduced basic object sample with $14<B<20$ (small grey dots). The previously known QSOs, Sey1s, and NELGs in the field are shown as in the first two panels a) for $B \leq 19.7$ and $\mathbf{b}$ ) for $19.7<B \leq 20$ ). In panel $\mathbf{c}$ ), the objects from Table 3 are indicated by $\bullet$. The symbols for QSOs with $z>2.2$ are framed. Panel d) shows the QSO candidates from the present study that were proved to be foreground stars (o) as well as the 10 medium-priority candidates without follow-up spectra $(+)$. The lines indicate the variability selection criteria (see text).

\subsection{Multi-object spectroscopy with TAUMOK}

The brighter candidates $(B<18)$ were observed with TAUMOK in the Schmidt focus of the Tautenburg $2 \mathrm{~m}$ telescope. TAUMOK allows to obtain simultaneously spectra of up to 35 objects within an area of 2.3 diameter (see Meusinger \& Brunzendorf 2001 for more details). The telescope was operated in a scanning mode prior to the spectroscopic observations in order to determine the most accurate positions of the fibres. The wavelength coverage is approximately 3800-9000 $\AA$, the reciprocal linear dispersion is $400 \AA \mathrm{mm}^{-1}$ corresponding to $9 \AA$ A per pixel.

The atmospheric conditions during the TAUMOK campaign were moderate. In five of the seven nights, spectra of
QSO candidates were taken. Four different fibre configurations were necessary to cover the VPM field. Several 1800 s exposures were taken for each configuration. The total exposure time per field is between 1.5 and 3 hours. Since the number of bright high-priority candidates is much smaller than the total number of available object fibres, most of the fibres were positioned at candidates of lower priority or on non-priority objects. Five fibres were reserved for template spectra from known QSOs with redshifts beteween $z=0.6$ and 2.5. Internal spectral lamps were used for the wavelength calibration prior and after the observation of a field. For the reduction of the TAUMOK spectra we applied a software package (Ball 2000) which is based on IRAF standard procedures for multi-object spectroscopy. 
Table 2. Observation log for the major spectroscopic follow-up observation runs.

\begin{tabular}{lccccc}
\hline \hline observing run & 1 & 2 & 3 & 4 & 5 \\
\hline spectrograph & TAUMOK & CAFOS & CAFOS & CAFOS & CAFOS \\
year/month & $1997 / 04$ & $1998 / 04$ & $1999 / 04$ & $2000 / 04$ & $2001 / 03$ \\
number of nights & 7 & 7 & 5 & 3 & 3 \\
number of objects & 41 & 46 & 34 & 23 & 35 \\
\hline
\end{tabular}

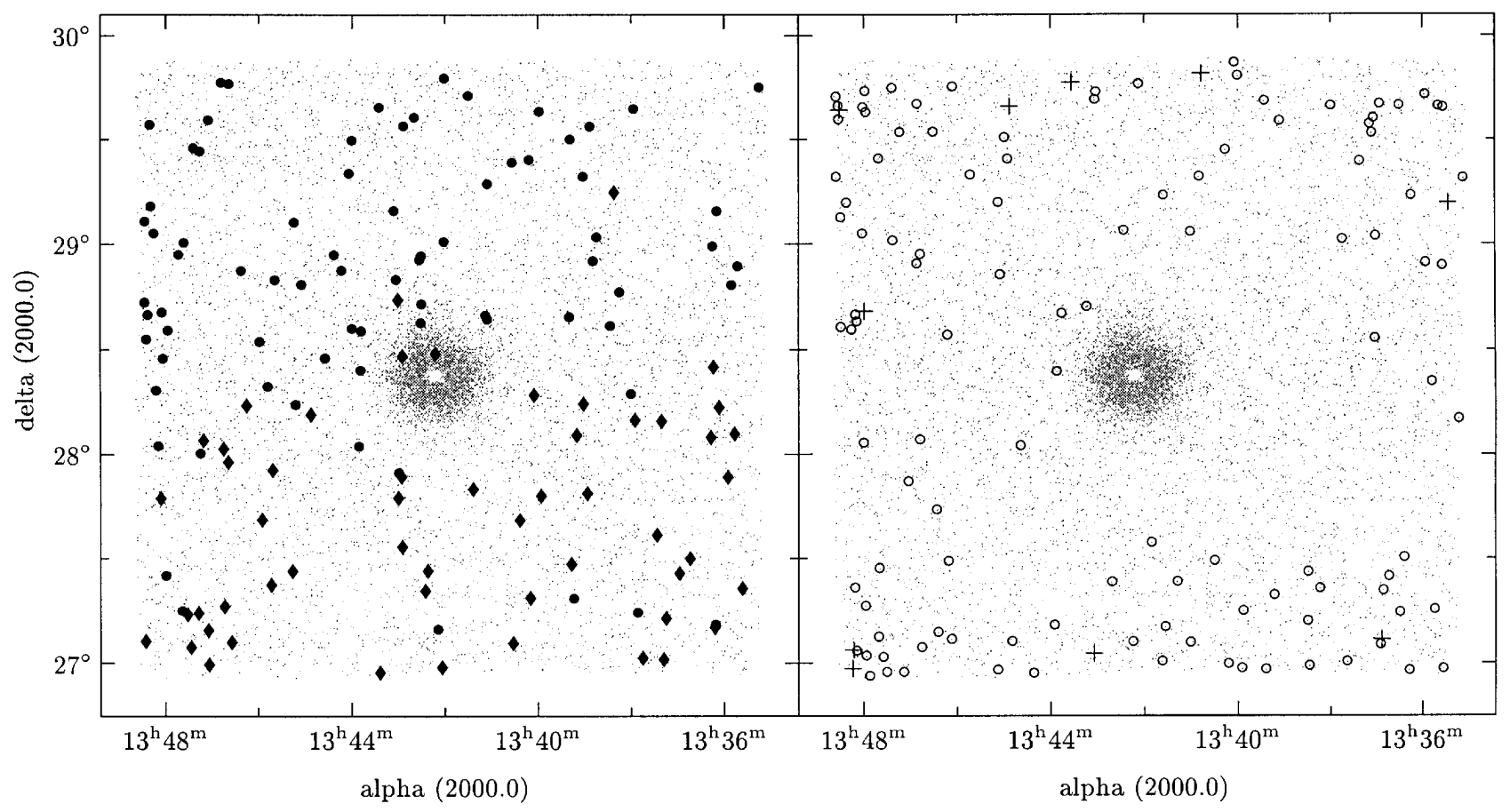

Fig. 3. Field of the VPM survey centred on the globular cluster M3. Star-like objects are shown as grey dots. The panel on the left hand side shows the distribution of the QSOs from the VPM search $(\bullet)$ as well as the QSOs with $B<19.7$ from the NED ( $\bullet$ ). The panel on the right hand side shows the distribution of the VPM candidates that were spectroscopically confirmed to be foreground stars $(\circ)$ as well as the medium-priority candidates without follow-up spectra (+).

\subsection{Single-object spectroscopy with CAFOS}

The candidates fainter than $B \approx 18$ were observed with the focal reducer and faint object spectrograph CAFOS at the Cassegrain-focus of the $2.2 \mathrm{~m}$ telescope of the German-Spanish Astronomical Centre on Calar Alto, Spain. The B 400 grism was used with a wavelength coverage of about 3000-9000 . The width of the entrance slit was adjusted to the seeing (typically 2-3 arcsec) resulting in an effective linear resolution of typically about $40-60 \AA$. Since the orientation of the long-slit was always North-South, some "slit-loss" due to atmospheric dispersion was unavoidable for spectra taken at hour angles significantly different from zero.

The total integration time varied between 10 and $90 \mathrm{~min}$, dependent primarily on the strength of the emission lines and on the weather conditions. Observations were made in a wide variety of atmospheric conditions. The weather was good in the 2001 observing campaign. In the previous runs, however, the fraction of observing time with good atmospheric transparency was rather low. Therefore, about half of all spectra have only a moderate signal-to-noise ratio. Several objects had to be observed in more than one run. Data reduction was performed using the long-slit spectroscopy package LONG of MIDAS. Wavelength calibration was done by means of calibration lamp spectra.

\subsection{Overview of the spectroscopically observed objects}

Spectra were taken for a total of 198 objects: 1.) In particular, all 54 high-priority candidates without classification in the NED were observed. 2.) Among the 95 medium-priority candidates, 17 objects have a measured redshift in the NED. For an additional 68 medium-priority objects spectra were taken in the framework of the present study. The remaining 10 objects have variabilities near the selection thresholds (Fig. 2d) and/or are located at the borders of the field (Fig. 3) where the contamination by foreground stars is obviously stronger (see below). The chance to find QSOs among these remaining 10 candidates is substantially lower than for the median-priority subsample as a whole. 3.) The number of low-priority candidates 


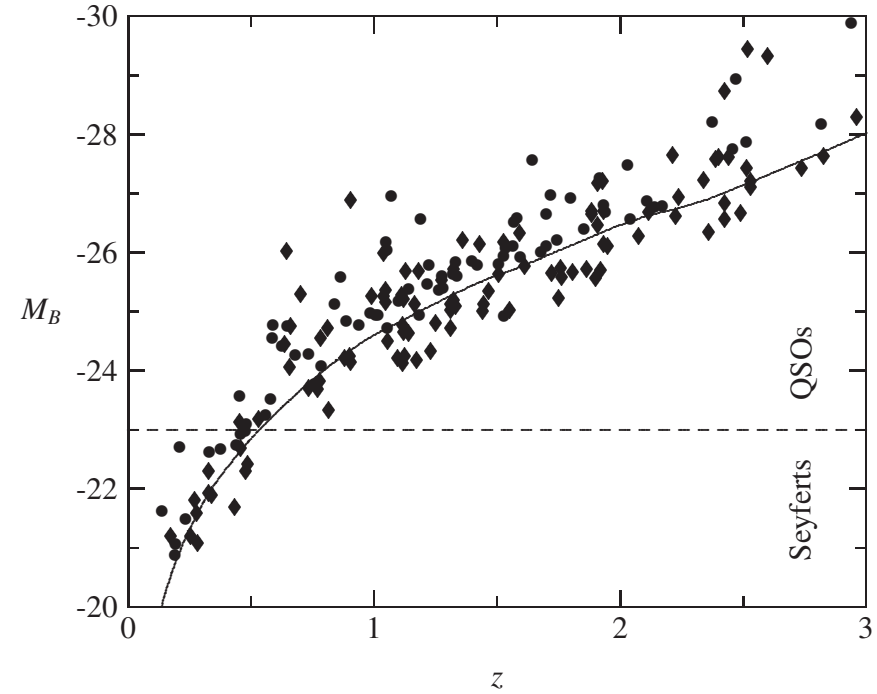

Fig. 4. Absolute magnitude $M_{\mathrm{B}}$ versus redshift $z$ for all known QSOs/Sey1s in the field identified with objects from our basic sample. The redshifts are either from the present study $(\bullet)$ or the NED $(\bullet)$. The continuous line indicates the magnitude limit $B \leq 19.7$ of the survey.

is obviously too large to reach a substantial completeness with regard to spectroscopic follow-up observations. We selected therefore from this priority class mainly the brighter objects $(18<B<19.5)$ and/or the objects with the strongest indication for variability. Note that the high fraction of QSOs/Sey1s among the observed low-priority objects is thus not representative for the whole subsample. There may be some undetected QSOs among the remaining low-priority objects, but their number is expected to be small.

The statistics of the observations for the various priority classes are summarised in Table 1 . Note that the total number of observed candidates listed there is smaller than the number of all observed objects given in Table 2. The reasons for this apparent discrepancy are the following: $(a)$ the criteria for the definition of priorities have slightly changed during the survey. (b) Many of the brighter objects observed with TAUMOK are not candidates in the sense of Table 1 , but were selected to allow a good positioning of the fibres. (c) Several objects were observed in more than one observing run. $(d)$ Since a variability survey is expected to be biased against low-variability QSOs, we selected also a few objects with quasar-typical colours, but with variability indices slightly below the selection thresholds. For instance, four objects were observed because they are X-ray sources. (e) A few strongly variable objects with $19.7<B<19.9$ have been observed as well.

\subsection{Source classification}

The spectral classification is based on the emission and absorption line properties. Three catagories are considered: (1.) redshifted broad emission lines and/or absorption lines, (2.) redshifted narrow emission lines, and (3.) unredshifted typical stellar absorption lines.
The first category comprises QSOs and Sey1s, which are discriminated by the usual luminosity threshold $M_{\mathrm{B}}=$ -23 . The absolute magnitude $M_{\mathrm{B}}$ is computed for $H_{0}=$ $50 \mathrm{~km} \mathrm{~s}^{-1} \mathrm{Mpc}^{-1}, q_{0}=0$ and the $K_{\mathrm{B}}$-correction from Brunzendorf \& Meusinger (2001). The data for the 69 QSOs and 5 Sey $1 \mathrm{~s}$ from the present study are listed in Table 3, and in the following, objects will be quoted with their number in this table. For most of these objects, redshifts were derived from several emission lines; in particular, strong narrow forbidden lines (e.g., [O III] 25007 ) were used, if present. The wavelength of a single line was measured by Gaussian centroids.

When we selected the first QSO candidate list in 1996, this list has been checked against the NED (cf. Paper I) to reject all objects already catalogued with measured redshifts. A new check revealed that six QSOs from Table 3 are catalogued in the February 2002 release of the NED. For three of these objects (No. 16, 68, 70) redshifts were published later than 1996. (Number 68 is identical with FBQS J1348+2840, White et al. 2000.) The other three (No. 29, 36, 42) had uncertain positions in the 1996 NED and therewith too large position differences (>10 arcsec) for an unambiguous identification. Further, we note that the QSO No. 11 is identified with the radio source (without $z$ in the NED) FIRST J133825.6+283637.

There are four narrow-emission line galaxies (NELGs) among the identified objects from the NED. An additional three NELGs were detected in the present study and are also listed in Table 4. The luminosities of the NELGs are clearly below the QSO-Sey1 threshold. In general, the class of the NELGs includes Seyfert 2s, narrow-line Seyfert 1s, LINERs, and $\mathrm{H}$ II galaxies. For this paper we have not attempted to separate the types of NELGs. One of the new NELGs (No. 43) is a high-priority QSO candidate, another one (No. 26) is of low priority, but with a high overall variability index $I_{\mathrm{var}}=2.45$. The third one (No. 15) has a high proper motion index and is not a QSO candidate from the VPM survey, but is one of the X-ray sources observed for completing the QSO sample. Among all objects with $z>0$ from our basic sample, No. 15 is the only one with a proper motion index significantly larger than the selection threshold $I_{\mathrm{pm}}=4$ (Fig. 1), perhaps indicating a wrong spectral classification from a noisy spectrum. All 7 NELGs were classified as star-like objects on the Schmidt plates; the infered redshifts are between 0.137 and 0.433 . In the frame of the VPM search in the M 92 field, a higher fraction of NELGs was detected due to a less stringent star-galaxy separation (Meusinger \& Brunzendorf 2001). The high variability indices measured for the NELGs were explained by increased photometric errors for objects with image profiles deviating from stellar ones (Meusinger \& Brunzendorf 2002).

Finally, an object is classified as a foreground star if its spectrum unambiguously shows typical un-redshifted stellar absorption lines. At a first glance, most of these objects are normal stars without unusual spectral features. Contrary to the QSOs, the classified stars show a remarkably inhomogeneous distribution over the field (Fig. 3): their strong concentration towards the outer parts and the corners of the field indicates an increase of the instrumental variability at large distances from the plate centre. Such an effect is in principle expected since we have not corrected for a position-dependence of the 


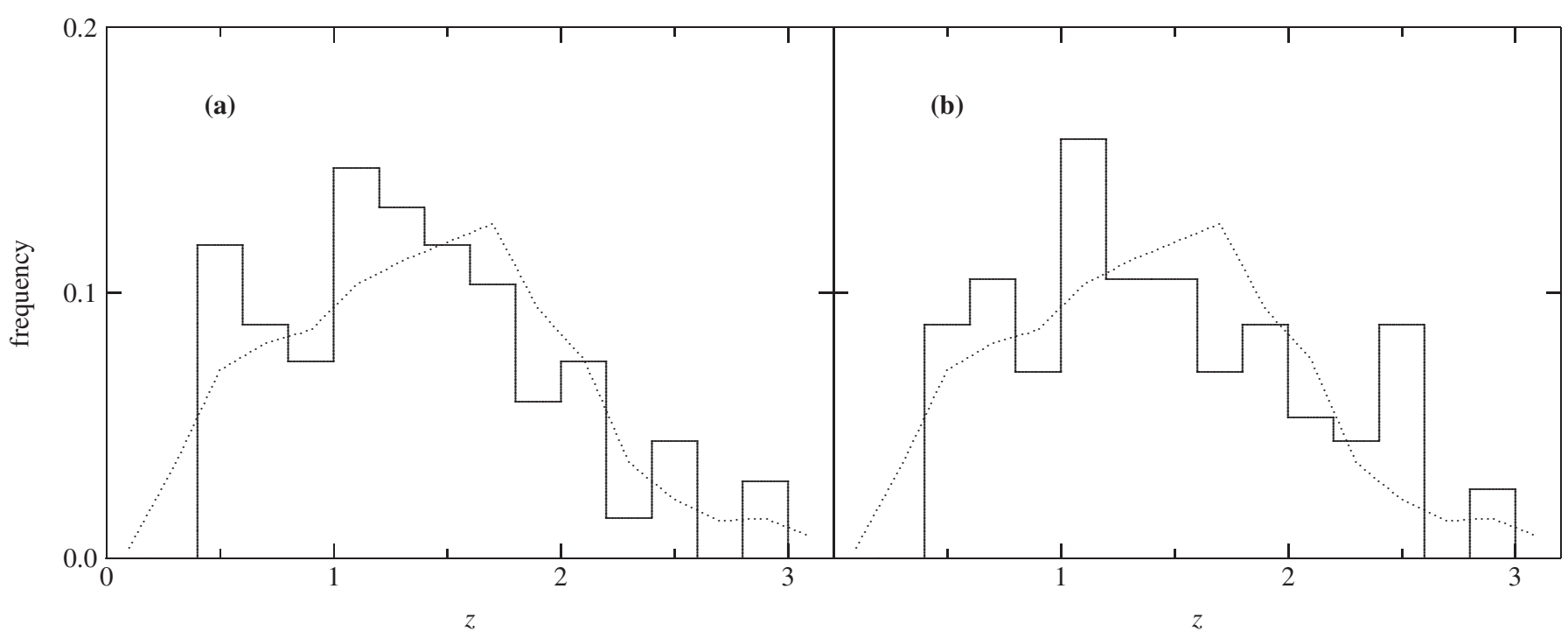

Fig. 5. Normalised distribution of the redshifts (number of QSOs per 0.2 redshift bin) for a) the subsample from the present follow-up spectroscopy (Table 3), and b) the (nearly) complete subsample of all 114 QSOs with $B \leq 19.7$. For comparison, the dotted polygon gives the normalized $z$ distribution for the QSOs from the SDSS Early Data Release (Schneider et al. 2002).

magnitude scale (PaperI). This interpretation implies that a substantial fraction of the selected stars are not really variables. In this context we note that most of the stars have lower variability indices than the QSOs (Fig. 2d).

To summarise, we have plausibly classified all 198 objects from our spectroscopic follow-up observations as either QSOs/Sey1s, NELGs, or foreground stars. There are new redshifts for 68 broad-lined objects and 3 narrow-lined objects. For an additional 6 already catalogued QSOs/Sey1s redshifts were confirmed.

\section{Properties of the QSO sample}

\subsection{General}

Table 3 lists redshifts, absolute magnitudes, colours, proper motion indices, and the two variability indices of the 77 QSOs, Sey1s, and NELGs from our follow-up spectroscopy. In a similar style, Table 4 summarises the data for the 104 QSOs, Sey1s, and NELGs identified in the NED. The distribution of these types over the three priority classes from the VPM survey is given in Table 4 . In the high priority subsample, $94 \%$ of the candidates were found to be QSOs/Sey1s, while the contamination by foreground stars is as low as $4 \%$. For the combined sample of high-and-medium-priority objects the success rate (i.e., the fraction of established QSOs/Sey1s among all candidates) is still as high as $63 \%$.

Figure 2 illustrates that a high fraction of all QSOs in the field are strongly variable. The $B$ standard deviation due to variability is about $0.2 \mathrm{mag}$ for QSOs with $B<19.7$. In this magnitude range, more than $60 \%$ of the QSOs/Sey1s show the strong variability of high-priority VPM candidates. (A detailed analysis of the variability properties will be deferred to a separate study.) For $90 \%$ of QSOs/Sey 1 s, both variability indices are above the selection thresholds. We find that 50 out of the $53 \mathrm{NED}$ QSOs/Sey1s with $B<19.7$ match the selection criteria of our survey, corresponding to a completeness

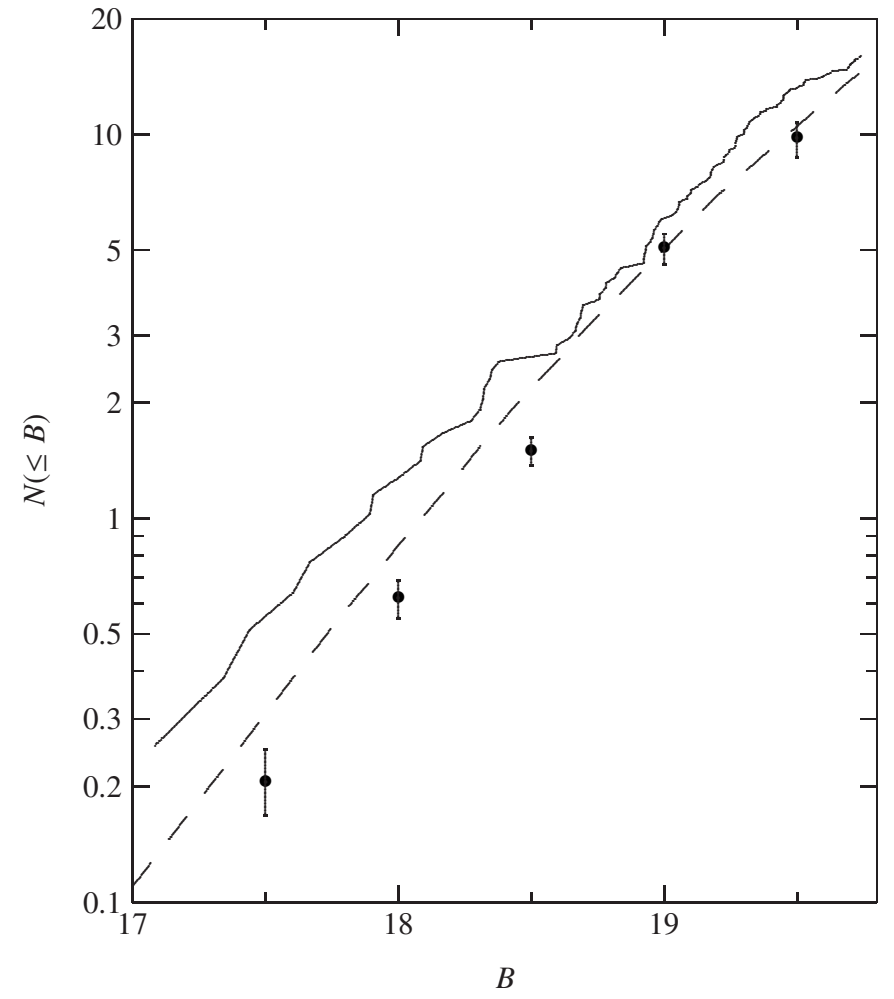

Fig. 6. Cumulative QSO surface density $N(\leq B)$, i. e. number of QSOs brighter than a given magnitude $B$ per square degree where $B$ has been corrected for an interstellar extinction of $A_{B}=0.05 \mathrm{mag}$. Solid polygon: all QSOs outside the cluster region $\left(d_{c}>24^{\prime}\right)$. Bullets with error bars: integral surface densities from Hartwick \& Schade (1990). The long-dashed curve corresponds to an analytical approximation given by Wisotzki (1998) that was derived from a composite optical QSO sample.

of $94 \%$ for the VPM survey. Only for two objects both variability indices fall below the selection thresholds; another one has a proper motion index slightly above the threshold. 

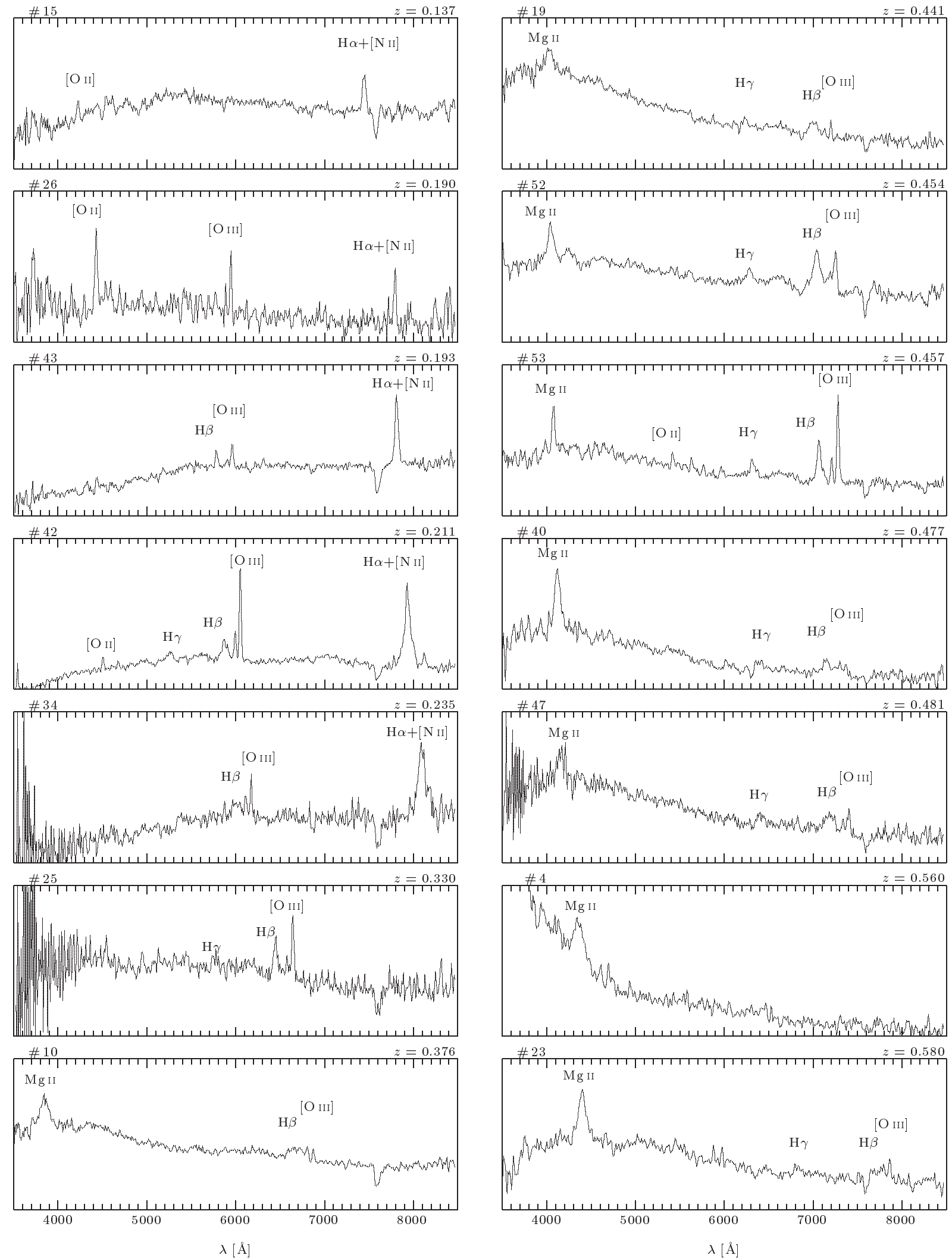

Fig. 7. Spectra (normalized flux $f_{\lambda}$ versus wavelength $\lambda$ ) of the QSOs, Sey1s, and NELGs from Table 3, sorted by increasing redshift. The running number from Table 3 and the redshift are given for each spectrum. Note that the spectra were not corrected for the atmospheric absorption bands at 6880 and $7620 \AA$.

The subsample of the 114 QSOs with $B<19.7$ is considered nearly complete. These QSOs are homogeneously distributed over the search field (Fig. 3). In particular, the QSO surface density in the northern half of the field, which is not covered by 
the CFHT blue grens survey, is comparable to that in the southern part where almost all known QSOs are in the CFHT survey. An additional three QSOs were detected by the VPM search in the CFHT field. Two of them (No. 5, 15) have "normal" spectra and were obviously ignored by chance in the CFHT survey; the other one (No. 7) shows very strong broad absorption line (BAL) features. The subsample is of course flux-limited, and $M_{\mathrm{B}}$ is therefore strongly correlated with $z$ (Fig. 4). Only for $z \leq 0.55$, the subsample is complete with regard to luminosities (since $B<19.7$ for QSOs of such $z$ ). Note that most of the objects with $z<0.55$ shown in Fig. 4 are Sey1s.

\subsection{Redshift distribution}

The redshift distribution is shown in Fig. 5 both for (a) the subsample from Table 3 and (b) the sample of all identified QSOs with $B \leq 19.7$. The shape of the $z$ distribution is roughly comparable with that from the SDSS Quasar Catalogue I. Early Data Release (Schneider et al. 2002), corroborating the result from VPM survey in the M92 field (Brunzendorf \& Meusinger 2002). This impression is confirmed by the two-tailed KS two-sample test. According to this test on a significance level $\alpha=0.05$, we have not to reject the null hypothesis that our subsamples (a) and (b) and the SDSS sample were drawn from the same population.

\subsection{Surface density}

The "completeness", or absolute efficiency, of the survey can be estimated by comparing the QSO surface densities, i.e. number counts per solid angle, to the densities predicted by other surveys. Figure 6 shows the surface density of all QSOs (i.e., $z>0, M_{\mathrm{B}}<-23$ ) with $B<19.7$ in our search field, compared with mean relations from various data samples. The cumulative density $N(<B)$ is simply computed by dividing the number of QSOs brighter than a given magnitude by the effective search area where the $B$ magnitudes were corrected for an interstellar extinction of $A_{\mathrm{B}}=0.05 \mathrm{mag}$. The size of the Schmidt field is $3.3 \times 3.3$. After subtracting the areas of the plate margins (not shown in Fig. 3), the calibration wedge, the crowded inner part of M 3 , and the area covered by the images of the objects in the remaining field, the effective search area amounts to $7.8 \mathrm{deg}^{2}$.

The resulting number-magnitude relation is roughly described by $\log N(<B) \propto x B$ with $x \approx 0.6$ for $17.5<B<18.5$ and $x \approx 0.75$ for $18.5<B<19.5$, in agreement with the result from the M92 field (Brunzendorf \& Meusinger 2002). The surface densities for our total QSO sample are higher than those derived by Hartwick \& Schade (1990), especially at brighter magnitudes. There are 9 QSOs with $B<18$ in

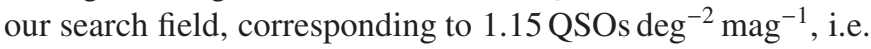
a factor of 1.8 more than in the Hartwick \& Schade data. More recently, La Franca \& Cristiani (1997) derived surface densities of $0.76 \mathrm{QSOs} \mathrm{deg}-20.5 \mathrm{mag}^{-1}$ for $17.9<B<$ 18.4, $0.3<z<2.2$, and $M_{\mathrm{B}}<-23$, to be compared

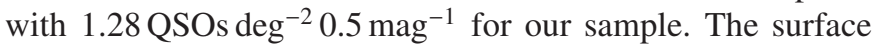
densities based on single-epoch observations are affected by variability and cannot be compared directly to those based on time-averaged magnitudes. It should be noticed however that Hartwick \& Schade corrected their data for such a variabilityinduced over-completeness. Note also that the different assumptions for $q_{0}$ (both Hartwick \& Schade and La Franca \& Cristiani adopted $q_{0}=0.5$ while we used $q_{0}=0$ ) make no significant difference for the number counts. We cannot exclude that the relative overabundance of apparently bright QSOs is due to the limitations of small-number statistics, but note that a similar result was found for the VPM survey in the M 92 field (Meusinger \& Brunzendorf 2001).

\subsection{Spectra}

The low-resolution spectra of the objects from Table 3 are shown in Fig. 7. The spectra are dominated by the typical AGNemission lines: $\mathrm{Ly} \alpha+\mathrm{N} v \lambda 1240$, Si Iv $+\mathrm{O}$ Iv] $\lambda 1400, \mathrm{C}$ Iv $\lambda 1549$, $\left.\mathrm{C}_{\mathrm{III}}\right] \lambda 1909, \mathrm{Mg}$ II $\lambda 2798$, [O $\left.\mathrm{mI}\right] \lambda \lambda 4959,5007$, and the Balmer series. A few objects (Nos. 10, 19, 64, 50, 11, 69, 7) apparently have relatively weak lines. Unfortunately, many of these spectra were taken at relatively bad atmospheric transparency, and poorly removed telluric lines may effect the equivalent widths of the QSO emission lines. Therefore we do not quantitatively discuss the distribution of the equivalent widths in this paper. The analysis of the QSOs from the VPM survey in the M92 field (where most of the spectra were taken under better weather conditions) has shown that the sampleaveraged line equivalent widths for the VPM QSOs are in good agreement with those from other samples of radio-quiet QSOs (Meusinger \& Brunzendorf 2001).

Broad absorption troughs are indicated in the spectra of the QSOs Nos. 3, 49, 37, 57, 7, 44, 75, 33, 65, and 70. For some other QSOs absorption features may be hidden due to the low signal-to-noise. The fraction of BAL QSOs is about $10 \%$, in good agreement with the BAL percentage in the SDSS Early Data Release (Schneider et al. 2002). There is only one object with an unusual spectrum: the BAL QSO No.7 where the emission lines are almost completely masked by extremely broad absortion lines. The best guess for the emission redshift is $z_{\mathrm{em}} \approx 1.7$, compared to $z_{\mathrm{abs}} \approx 1.5$ for the strongest absorption lines (C Iv $\lambda 1549, \mathrm{Al}$ III $\lambda 1860$, and the Fe II-multiplet at 22500). This object is a high-priority VPM QSO candidate with quite red colours (see below). There is no entry in the NED at this position. Objects like No. 7 are not very likely to be recognized by most other optical QSO surveys. For a few other QSOs/Sey1s, the spectra in Fig. 7 have unusually red continua (Nos. 42, 64, 48, 3). However, the $U-B$ indices (Table 3 ) of these objects closely follow the mean colourredshift relation (Fig. 9) and thus the missing blue light in the spectra is interpreted by the slit-loss effect due to atmospheric dispersion (Sect. 1). We conclude that, up to the limit of the survey, the fraction of QSOs with unusual spectra is at maximum a few percent. This conclusion is again in agreement with the statistics from the (still incomplete) SDSS data (Hall et al. 2002). 


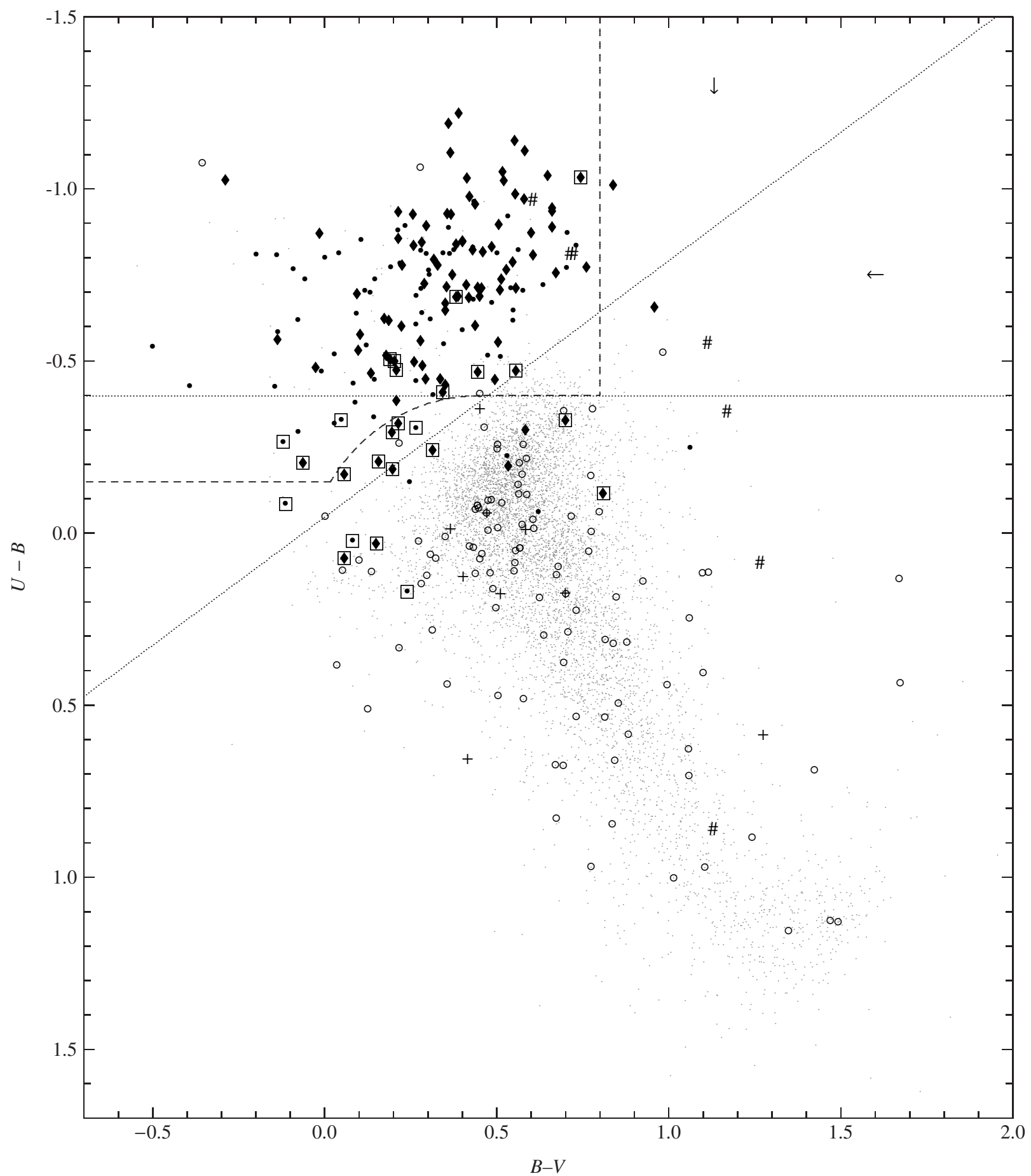

Fig. 8. Colour-colour diagram for the M 3 field. QSOs/Sey 1s are shown as • (present study) or $\bullet$ (CFHT survey), respectively. Quasars with $z>2.2$ are framed with a $\square$. The arrows on the right and at the top, respectively, indicate QSOs with unknown $B-V$ and $U-B$ colours, respectively. The \# symbols indicate narrow emission line galaxies. Open circles are VPM-QSO candidates that were spectroscopically identified as foreground stars; medium-priority candidates without spectroscopic follow-up observations are shown as plus signs. Other star-like objects with $14<B<20$ are shown as small dots. Selection criteria from colour surveys are indicated by horizontal dotted line: UVX search, diagonal dotted line: two-colour search as discussed in Paper I, dashed curve: two-colour selection according to LaFranca et al. (1992).

\subsection{Colour indices}

In Paper I, a colour-colour diagram of the QSO candidates was presented showing a broad scatter of their colour indices and a large fraction of red QSO candidates. The distribution of the spectroscopically classified objects on the $U-B$ versus $B-V$ plane is shown in Fig. 8. The most important result is the fact that all candidates with extremely red colours proved to be foreground stars, in agreement with what we found from the VPM survey in the M92 field. Obviously, the QSOs from Table 3 populate essentially the same area as the QSOs from the CFHT 

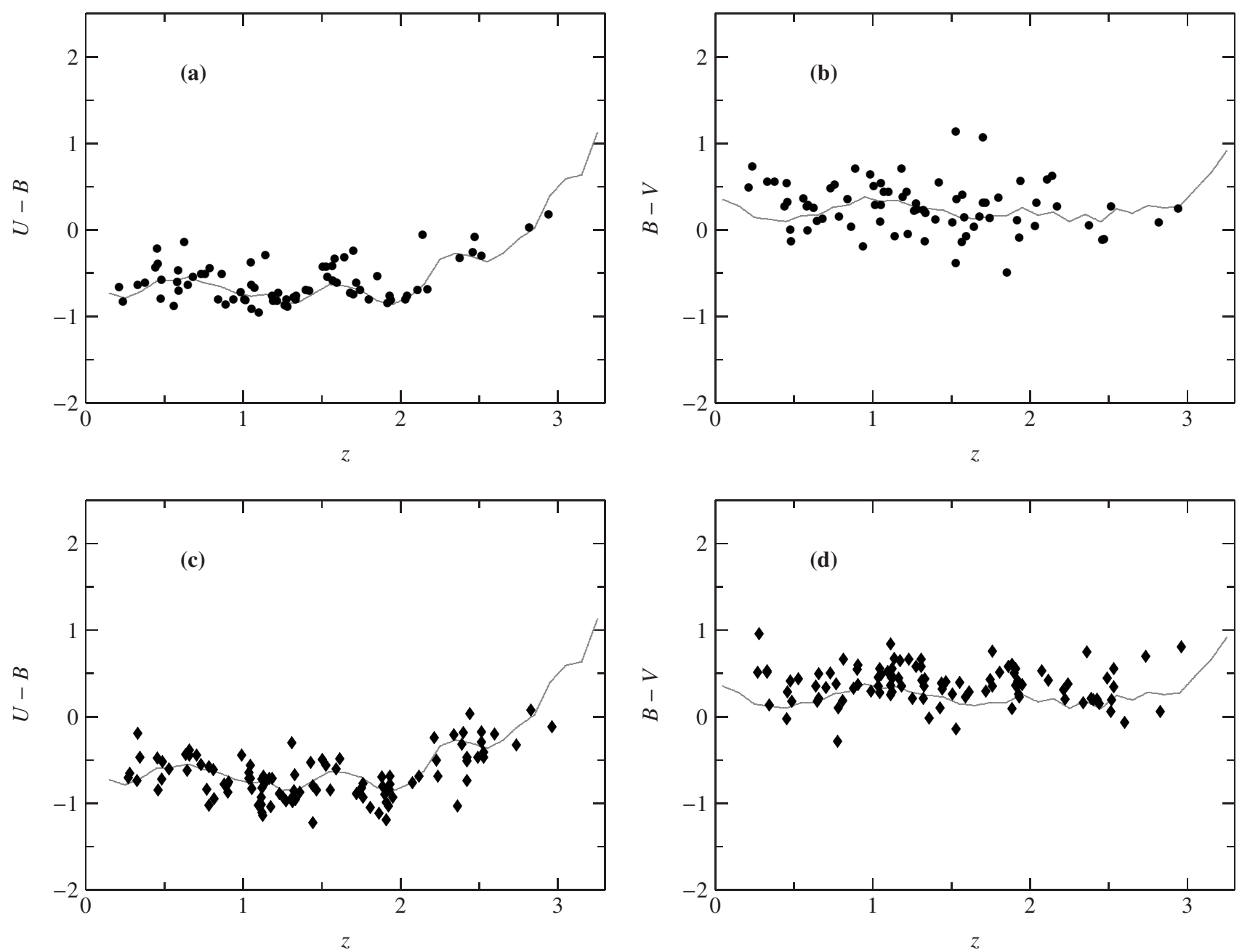

Fig. 9. Colour-redshift relations for the the QSOs/Sey1s in the M3 field. Panels a), b) show the objects from Table 3 (•). Panels c), d) show the QSOs/Sey1s from the NED identified with objects from our list ( $\downarrow)$. For comparison, the mean relations for the QSOs from Véron-Cetty \& Véron (2001) are plotted.

grens survey. For $z<2.2$ this area is well defined by the selection criteria of classical colour surveys. There are only 7 low-redshift $(z<2.2)$ QSOs located beyond the demarcation line for colour selection discussed in Paper I. A typical fluctuation of about $0.35 \mathrm{mag}$ per colour index is expected due to photometric errors and variability (as the time-lines for the three colour bands are not identical). In addition, a scatter may be produced by intrinsic differences in the continuum slope and/or the strength of emision lines and/or absorption troughs. Remarkably, the strongest deviation from the colour selection line is measured for the two absorption line QSOs Nos. 7 and 75.

The same conclusion is reached from the colour-redshift relations (Fig.9). Apart from the scatter due to variability and photometric errors, the QSOs from our sample closely follow the mean relation of QSOs from the Véron-Cetty \& Véron (2001) catalogue. Among the QSOs from Table 3, the strongest deviation is measured again for Nos. 7 and 75. The QSO No.28, which is the faintest object in Table 3, shows a strong deviation in $B-V$. Fainter QSOs tend to have larger colour indices $B-V$ (Fig. 9d).
Data from the 2 Micron All Sky Survey (2MASS; Skrutskie et al. 1997), March 2000 data release are available for $25 \%$ of the field. With an identification radius of 10 arcsec we identified six QSOs from our whole sample with catalogued 2MASS sources. For all six objects the $B-K_{\mathrm{s}}$ colour index is smaller than 4 , i.e. smaller than for the red QSOs found by Webster et al. (1995) among the flat-spectrum radio-loud QSOs.

For the M92 field we have estimated that the fraction of QSOs with unusually red $B-V$ colour indices must be less than $3 \%$ up to $B=19.8$ (Brunzendorf \& Meusinger 2002). From the data in the M3 field we estimate a similar fraction of about $2 \%$ up to $B=19.7$. Although a survey in the $B$-band is obviously not an ideal approach to derive strong conclusions about the underlying population of possibly highly reddened QSOs, the unbiased VPM QSO sample provides a constraint of its properties. Let us assume for simplicity that there are two QSO populations of comparable size: normal QSOs and reddened QSOs with an intrinsic dust reddening equivalent to $E_{B-V}=0.5$ - a not unreasonable level in a dusty system - implying an extinction of about 2 mag in the $B$-band (assuming a galactic extinction curve). Using the 
number-magnitude relation from Fig. 6, we would expect to detect about 5-7 strongly reddened QSOs up to $B_{\lim }=19.7$ in each search field. This is clearly more than what we found, indicating that the red QSOs are either redder on average or less frequent. A more detailed discussion of this question has to be deferred to a separate study.

\subsection{QSO pairs}

The search for pairs with an angular separation of up to 2 arcmin yields 8 combinations, but the redshift differences are very large for 7 of them. The closest pair in the threedimensional space consists of Nos. 38 and 40 from Table 4 with $z=1.310$ and 1.325 and an angular distance of 96 arcsec. For objects with small separations (e.g., <10 arcsec), the measurements of variability and proper motion are attended with additional uncertainties. Such objects are, in principle, rejected from the VPM candidate list. This does not significantly reduce the general efficiency of the search method but the efficiency of the detection of close pairs.

\section{Conclusions}

We performed a VPM QSO survey with a limiting magnitude of $B_{\lim } \approx 20$ in a 10 square degrees field at high galactic latitude. The VPM technique proved to be an efficient method for finding QSOs. As the result of the spectroscopic followup observations of 198 candidates and the identification of further candidates in the NED, a sample of 175 QSOs/Sey1s with $0.4<z<3$ is available. With a stellar contamination of only about 4\%, the high-priority QSO subsample from the VPM search is very clean. For the combined sample of high-and-medium-priority objects the fraction of established QSOs/Sey1s among all candidates is still as high as $63 \%$. The completeness of the VPM QSO sample with $B \leq 19.7$ is estimated to be $94 \%$. The number-magnitude relation for that sample is in good agreement with the one expected from the relation derived by Wisotzki (1998) from various QSO samples. At brighter magnitudes ( $B<18.5$ ), we find a somewhat higher QSO surface density.

The optical broad-band colours and the spectra of the VPM-selected QSOs are not significantly different from those of QSOs selected by other optical surveys, in agreement with what we found in the M92 field (Meusinger \& Brunzendorf 2001; Brunzendorf \& Meusinger 2002). Such a result can not be a priori expected since the selection criteria of the VPM survey are completely different from those in most other optical surveys. Although there is a large fraction of objects with red colours among the VPM QSO candidates, all candidates with extremely red colours were proved to be stellar contaminants. We estimate that the fraction of QSOs with unusualy red optical colours is at most a few per cent up to the limit of the survey, provided that their variability properties are not significantly different from those of the other QSOs. Some BAL QSOs are known to be considerably redder than the targets of most QSO surveys (e.g., Weymann et al. 1991; Menou et al. 2001; Hall et al. 2002). The fraction of such unusual QSOs in the (incomplete) SDSS Early Data Release is less than $1 \%$
(Hall et al. 2002), in good agreement with our result. In this context it is notable that all VPM QSOs with indication for substantial absorption are strongly variabel $\left(I_{\mathrm{var}}>1.5\right)$. A VPM search is thus expected to be essentially unbiased against strongly absorbed QSOs, apart from the bias introduced by the band-pass of the search. The general agreement of the properties of the VPM QSO sample with those from more conventional optical surveys suggests that the latter do obviously not ignore a substantial number of red QSOs up to $B \approx 20$. On the other hand, we can conclude that the VPM survey can be combined with colour search criteria in order to reach a very high efficiency without a significant loss of completeness. Of course, we can not exclude the existence of substantial numbers of obscured red QSOs that are fainter than the current survey limit. Such objects can be found by a deeper VPM survey.

Acknowledgements. This research is based on observations made with the $2.2 \mathrm{~m}$ telescope of the German-Spanish Astronomical Centre, Calar Alto, Spain. This research has made use of the NASA/IPAC Extragalactic Database (NED) which is operated by the Jet Propulsion Laboratory, California Institute of Technology, under contract with the National Aeronautics and Space Administration.

\section{References}

Antonucci, R. 1992, ARA\&A, 31, 473

Ball, M. 2000, Ph.D. Thesis, University Jena

Becker, R. H., White, R. L., Gregg, M. D., et al. 2000, ApJ, 538, 72

Becker, R. H., Gregg, M. D., Hook, I. M., et al. 1997, ApJ, 479, L93

Bershady, M. A., Trevese, D., \& Kron, R. G. 1998, ApJ, 496, 103

Boyle, B. J., Fong, R., Shanks, T., et al. 1990, MNRAS, 243, 1

Brunzendorf, J., \& Meusinger, H. 2001, A\&A, 373, 38

Brunzendorf, J., \& Meusinger, H. 2002, A\&A, 390, 879

Conti, A., Kennefick, J. D., Martini, P., et al. 1998, AJ, 117, 645

Crampton, D., Cowley, A. P., \& Hartwick, F. D. A. 1990, AJ, 100, 47

Croom, S. M., Smith, R. J., Boyle, B. J., et al. 2001, MNRAS, 322, L29

Francis, P. J., Whiting, M. T., \& Webster, R. L. 2000, Publ. Astron. Soc. Aust., 53, 56

Goldschmidt, P., \& Miller, L. 1998, MNRAS, 293, 107

Gregg, M. D., Lacy, M., White, R., et al. 2002, ApJ, 564, 133

Hall, P. B., Anderson, S. F., Strauss, M. A., et al. 2002, ApJS, in press [astro-ph/0203252v1]

Hartwick, F. D. A., \& Schade, D. 1990, ARA\&A, 28, 437

Hasinger, G., Burg, R., Giacconi, R., et al. 1998, A\&A, 329, 482

Hawkins, M. R. S. 1983, MNRAS, 202, 571

Hewett, P. C., Foltz, C. B., \& Chaffee, F. H. 1995, AJ, 109, 14, 98

Hook, I. M., McMahon, R. G., Boyle, B. J., et al. 1994, MNRAS, 268, 305

La Franca, F., Cristiani, S., \& Barbieri, C. 1992, AJ, 103, 1062

Kron, R. G., \& Chiu, L.-T. 1981, PASP, 93, 397

La Franca, F., \& Cristiani, S. 1997, AJ, 113, 11517

Lamer, G., Brunner, H., \& Staubert, R. 1997, A\&A, 327, 467

Maiolino, R., Salvati, M., Marconi, A., et al. 2001, A\&A, 375, 25

Majewski, S. R., Munn, J. A., Kron, R. G., et al. 1991, in The Space Distribution of Quasars, ed. D. Crampton, ASP Conf. Ser., 21, 55

Menou, K., Vanden Berk, D. E., Ivecić, Ž., et al. ApJ, 561, 645

Meusinger, H., Klose, S., Ziener, R., \& Scholz, R. D. 1995, in The Future Utilisation of Schmidt Telescopes, Proc. of IAU Coll. 148, ed. J. M. Chapman, R. Cannon, S. Harrison, \& B. Hidayat, ASP Conf. Ser., 84, 486 
Meusinger, H., Brunzendorf, J., Scholz, R.-D., et al. 1997, in Treasure Hunting in Astronomical Plate Archives, ed. P. Kroll, C. la Dous, H.-J. Bräuer, et al. (Thun \& Frankfurt: Harri Deutsch), 122

Meusinger, H., \& Brunzendorf, J. 2001, A\&A, 374, 878

Meusinger, H., \& Brunzendorf, J. 2002, A\&A, 390, 439

Meusinger, H., Brunzendorf, J., Scholz, R.-D., et al. 2002, in ASP

Conf. Ser., AGN Surveys, Proc. of IAU Coll. 184, ed. R. F. Green,

E. Ye. Khachikian, \& D. B. Sanders, in press

Risaliti, G., Marconi, A., Maiolino, R., et al. 2001, A\&A, 271, 37

Sanders, D., Soifer, B. T., Elias, J. H., et al. 1988, ApJ, 325, 74

Schneider, D. P., Richards, G. T., Fan, X., et al. 2002, AJ, 123, 567

Scholz, R.-D., Meusinger, H., \& Irwin, M. 1997, A\&A, 325, 457 (Paper I)

Sharp, R. G., McMahon, R. G., Irwin, M., et al. 2001, MNRAS, 326, 45
Skrutskie, M. F., Schneider, S. E., Stiening, R., et al. 1997, in The Impact of Large Scale Near-IR Sky Surveys, ed. F. Garzon, N. Epchtein, A. Omont, et al. (Dordrecht: Kluwer Academic Publishing Company), 25

Véron, P., \& Hawkins, M. R. S. 1995, A\&A, 296, 665

Véron-Cetty, M. P., \& Véron, P. 2001, Quasars and Active Galactic Nuclei (10th ed.), A\&A, 374, 92

Webster, R. L., Francis, P. J., Peterson, B. A., et al. 1996, Nature, 375, 469

Weymann, R. J., Morris, S. L., Foltz, C. R., et al. 1991, ApJ, 373, 23

White, R. L., Becker, R. H., Gregg, M. D., et al. 2000, ApJS, 126, 133

Wisotzki, L. 1998, Astron. Nachr., 319, 5

Wisotzki, L., Christlieb, N., Bade, N., et al. 2000, A\&A, 358, 77 